\title{
Anaesthesia for a Patient with Rubinstein Taybi Syndrome
}

\author{
Harshika C. A. Hannadige ${ }^{1^{*}}$, Senaka Weerakoon ${ }^{2}$ \\ ${ }^{1}$ Registrar in Anaesthesia, ${ }^{2}$ Consultant Anaesthetist, Lady Ridgeway Hospital for Children, \\ Colombo, Sri Lanka
}

\begin{abstract}
Cases of anaesthetic management in Rubinstein Taybi Syndrome (RTS) has been confined to a small number since it was first diagnosed in 1963. Anaesthesia for RTS is rare, but consists of few important anaesthetic implications. We present a 3year-old boy with RTS who underwent general anaesthesia for correction of clinodactyly. He experienced an episode of desaturation with no obvious reason that lead him to have supplemental oxygen and monitoring in the intensive care unit.
\end{abstract}

Key words: Rubinstein Taybi Syndrome, anaesthesia, airway

\section{Introduction}

Rubinstein-Taybi Syndrome or Broad ThumbHallux syndrome was first described by Michail et al. in $1957 .{ }^{1}$ A larger constellation of features was described by Jack Herbert Rubinstein and Hooshang Taybi in $1963 .^{2}$

RTS is an uncommon disorder characterized by high arched palate, broad thumbs, high arched eyebrows, hirsutism, beaked nose with flat bridge, abnormal ears and mental retardation. Other associations include microcephaly, short stature, defects in eyes, deviated nasal septum, cryptorchidism, abnormalities in fingers and toes and hyper extensible joints. ${ }^{3}$ The prevalence of RTS is around 1 in 300,000 in the general population. RTS happens as a consequence of submicroscopic 16p13.3 deletion of the response element binding protein (CREBBP). ${ }^{4}$

*Correspondence: Harshika C. A. Hannadige

E mail: harshikahannadige@ymail.com

https://orcid.org/0000-0002-0050-2989

Received: 13/11/2020

Accepted: 22/08/2021

DOI: https://doi.org/10.4038/slja.v29i2.8715

\section{Case Report}

A 3-year-old, $13 \mathrm{~kg}$ boy with RTS was scheduled for bilateral thumb clinodactyly correction. He was otherwise healthy. He had two previous hospital admissions to undergo laparoscopic orchidopexy under general anaesthesia on two different occasions. Past anaesthetic history was unremarkable. He was born at term by lower segment caesarean section as his mother had gestational diabetes mellitus. He had one episode of neonatal hypoglycaemia on day one that was treated. He was diagnosed with RTS in the first week after birth. He attained motor development according to his chronological age but speech was delayed. $\mathrm{He}$ did not have any cardiac abnormality.

Physical examination revealed characteristic features of RTS including short stature, beaked nose, hirsutism, microcephaly and hypertelorism. Informed written consent for anaesthesia and surgery was taken from the parents. He was kept fasted 6 hours for solids and 2 hours for clear liquids.

Gas induction was done with sevoflurane and oxygen. ECG, $\mathrm{SpO}_{2}$ and non-invasive blood pressure monitoring was started. A 22G intravenous cannula was inserted. He was given fentanyl and after achieving adequate depth of anaesthesia direct laryngoscopy was performed and it was grade 1 as per Cormack and Lehane 
grading. $2 \%$ lignocaine $1 \mathrm{ml}$ was sprayed to vocal cords to obtund the intubation response. He was intubated with an uncuffed size $4.0 \mathrm{~mm}$ endotracheal tube and the tracheal tube placement was confirmed clinically as well as by capnography.

Anaesthesia was maintained with sevoflurane, oxygen and air. The surgeons gave bilateral ring blocks to thumbs with $0.25 \%$ plain bupivacaine. Rectal paracetamol was inserted. The surgery lasted for about two hours and ventilation was supported using a Mapleson F circuit. His maintenance fluid requirement was given intraoperatively. He had an unremarkable intraoperative period.

After surgery, he was extubated when he became fully awake. Despite having good breathing efforts his oxygen saturation on room air began to drop to around $86 \%$. Possible causes of desaturation such as upper airway obstruction, laryngospasm, bronchospasm and residual anaesthetic effect were considered, but there was no plausible cause for the desaturation. He could maintain saturation around $97 \%$ with $2 \mathrm{~L} / \mathrm{min}$ of oxygen via nasal prongs. He was admitted to ICU for oxygen therapy and post-operative observation. He was off oxygen the next day and sent to ward. He was discharged home the same evening.

\section{Discussion}

Patients with RTS may require surgery for multiple defects. They include correction of craniofacial, bone, orthodontic, genitourinary, eye and cardiac lesions. Anaesthesia for a patient with RTS is quite challenging because of the limited availability of evidence.

The craniofacial abnormalities may render laryngoscopy and intubation difficult especially when there is associated micrognathia, high arched palate and limited mouth opening. Though we were able to successfully intubate with direct laryngoscopy, it is recommended to demonstrate the ability to bag and mask ventilate before administering the neuromuscular blocking agent. The craniofacial abnormalities may contribute to and worsen obstructive sleep apnoea. Some patients have been found to have congenital laryngeal or tracheal stenosis under anaesthesia. Therefore, meticulous preparation for a difficult airway is of utmost importance.

Perioperative respiratory and ventilatory problems can be further complicated by abnormal lobulations in the lungs, copious mucous secretions and gastro-oesophageal reflux. Excessive secretions have been noted by other authors that may make inhalational induction troublesome and the immediate postoperative period complicated. This might be the probable reason why this patient desaturated post-operatively. It is prudent to monitor oxygen saturation post-operatively in an ICU for more complex surgeries considering the potential airway problem.

About one third of patients with RTS have congenital heart disease, which include atrial septal defect (ASD), ventricular septal defect (VSD), patent ductus arteriosus (PDA), coarctation of aorta, pulmonic stenosis, bicuspid aortic valve and hypoplastic left heart syndrome. In addition to these structural cardiac defects, conduction defects have also been reported, particularly in association with the use of succinylcholine, atropine and neostigmine. There are reports of arrhythmias following the use of succinylcholine and therefore it is better avoided. Although non-depolarizing muscle relaxants have been used, the safest method would be intubation without using any muscle relaxant, especially when the patient is hypotonic, as done in our patient with sevoflurane, fentanyl and local anaesthetic.

In summary, there are many concerns with regards to anaesthesia in a child with RTS. Airway difficulties, respiratory disabilities and cardiac lesions are of primary concern. 


\section{References}

1. Michail J, Matsoukas J, Theodorou S, "Arched, clubbed thumb in strong abduction-extension \& other concomitant symptoms." Revue de Chirurgie Orthopédique et Réparatrice de l'Appareil Moteur, vol. 43, no. 2, pp. 142-146, 1957.

2. Rubinstein JH, Taybi H. Broad thumbs and toes and facial abnormalities. A possible mental retardation syndrome. Am J Dis Child. 1963; 105:588-608

3. 3. Mc Arthur RG. Rubinstein Taybi syndromes: broad thumbs and great toes, facial abnormalities and mental retardation. A presentation of three cases. Can Med Assoc J.1967; 96(8):462-466

4. 4. Breuning $\mathrm{MH}$, Dauwerse HG, Fugazza G, et al. Rubinstein- Taybi syndrome caused by submicroscopic deletions within 16p13.3 Am J Hum Genet. 1993; 52(2):249-254 